\title{
MHYRO: Modular HYbrid RObot for contact inspection and maintenance in oil \& gas plants
}

\author{
A. Lopez-Lora, P.J. Sanchez-Cuevas, A. Suarez, A. Garofano-Soldado, A. Ollero and G. Heredia
}

\begin{abstract}
In this paper, we propose a new concept of robot which is hybrid, including aerial and crawling subsystems and an arm, and also modular with interchangeable crawling subsystems for different pipe configurations, since it has been designed to cover most industrial oil \& gas end-users' requirements. The robot has the same ability than aerial robots to reach otherwise inaccessible locations, but makes the inspection more efficient, increasing operation time since crawling requires less energy than flying, and achieving better accuracy in the inspection. It also integrates safety-related characteristics for operating in the potentially explosive atmosphere of a refinery, being able to immediately interrupt the inspection if a hazardous situation is detected and carry the sensible parts such as batteries and electronic devices away as soon as possible. The paper presents the design of this platform in detail and shows the feasibility of the whole system performing indoor experiments.
\end{abstract}

Index Terms-Aerial Systems: Applications;

\section{INTRODUCTION}

A ERIAL robots are becoming increasingly popular in recent years since their wide variety of applications is growing day by day [1][2][3][4]. These applications range from cargo transport [5] infrastructure inspection [6][7], cinematography [8] to search and rescue [9][10] in post-disaster situations. Nowadays, there are many industries that consider them as a disruptive technology that will change the future of several applications. A clear sign of this is the attention of industries related to inspection and maintenance with drones [11] in oil and gas plants, wind turbines [12], bridges [13] or power lines [14][15] are paying to these aerial robots. The reason that motivates this interest is the ability that these robots have to reach quickly high altitude workspaces, work in large outdoors scenarios and, of course, avoiding the presence of humans in hazardous workspaces, preventing accidents. However, although in these previous works aerial robots accomplish inspection tasks in industrial environments, like chemical or solar plants, large infrastructures, such as bridge and tunnels, and even power lines, in general the proposed solutions are significantly limited in terms of flight time and quality of the measurements. Moreover, the variety of scenarios that can be found in a real industry hinder the suitability of those

A. Lopez-Lora (abrloplor@alum.us.es), $\quad$ P. Sanchez-Cuevas (psanchez16@us.es), A. Suarez (asuarezfm@us.es), A. Garofano-Soldado (ambgarsol@alum.us.es), G. Heredia (guiller@us.es), and A. Ollero (aollero@us.es) are with the GRVC Robotics Lab of Seville, Spain.
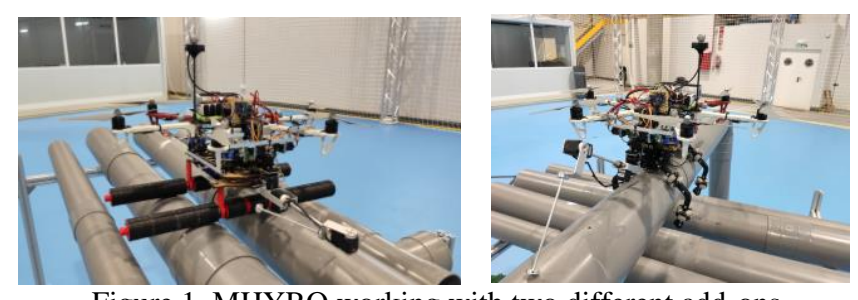

Figure 1. MHYRO working with two different add-ons aerial robots. In general, these drawbacks are directly derived from the use of general purpose platforms, which have many capabilities but have not been designed to solve the specific problems of the industry.

In particular, oil and gas industrial plants and refineries usually have thousands of kilometers of pipes that have to be periodically inspected, measuring wall thickness with ultrasonic sensors to detect corrosion and prevent spills. These pipes have diameters ranging from 6 to 24 inches and come in a large variety of configurations, from isolated pipes to racks of multiple pipes with different or no separation, with elbows and all sort of connections. In addition, several measurements have to be done in each pipe section, so the inspection time in each part can be significant. So, a hybrid robot able to fly to the inspection area and land and move on the pipe during the inspection is an energy-efficient solution.

Robots that combine different mobility systems have been designed to work in complex environments. These include aerial robots that are able to perch on walls and cables [16][17][18][19], prototypes that fly and roll over ground [20], and also robots that fly and sail on water [21] or can fly and dig underwater [22]. However, most of these prototypes have been built for multimodal mobility demonstration and have not been designed to perform work in industrial plants.

Furthermore, most parts of a refinery have a potentially explosive atmosphere, and then any machine working inside should be ATEX certified. Since this is extremely difficult to achieve by an aerial robot, what is known as a hot-work permit has to be used. This is a special per-operation authorization with rigorous safety measures that require to have gas sensors onboard the aerial robot and in the surroundings, and strict emergency procedures in case of gas detection, that demand the robot to leave the area in few seconds taking with it all ignition sources (i.e. batteries).

In this work, we propose a modular hybrid aerial robot, MHYRO (see Figure 1), which has been specifically designed for pipe inspection in the oil and gas industry. The robot has an energy-efficient hybrid configuration because it 
is able to fly and also land and crawl over the pipes and, it is modular because it has been designed with interchangeable landing gears which will be mounted depending on the application scenario. In this paper, two landing gears have been included to be able to land over a pipe or a rack of pipes. Moreover, the robot has a robotic arm which, once landed, is deployed to carry out the inspection of the pipe measuring, for example, the thickness underside. Last, the docking system which connects the landing gear and the aerial platform is also an emergency system which, combined with a gas sensor, is able to detach the landing gear and the robotic arm flying away with the batteries and the electronic systems if a hazard situation is detected in an explosive environment.

The rest of the paper is structured as follows: Section II describes and justifies the robot concept including a brief description of the problem to solve. Section III details the mechanism of the different robotic parts of the robot as well as the interfaces between them. Section IV shows the control architecture required to perform a fully autonomous mission. Section V presents and validates the suitability of the system proposed to accomplish the pipe inspection task. Moreover, it presents a brief coverage analysis of the system, differentiating the flight, inspection and operation times. Conclusions and future works are presented in Section VI.

\section{SYSTEM DESCRIPTION}

\section{A. Problem Statement}

The proposed system has been designed aiming to pipe inspection by contact applications. The experience of the authors in similar applications has demonstrated that the use of UAVs can change the maintenance programs of the oil and gas industries in a disruptive way. Moreover, in those previous projects [23][24] the authors also noted that endusers need high accuracy in the inspections, which is very difficult to achieve while the platform is flying. This is because the movement of the aerial platform disturbs the inspection results, for example, caused by vibrations in the aerial vehicle or bounces in the contact point. Thus, the robotic platform presented in this paper follows those recommendations, in such a way that the inspection phase is only carried out when the aerial platform is landed or perched in the pipe and is stabilized over it.

Furthermore, the system aims to be an ad-hoc solution to the use case of an oil and gas refinery. Since these environments are very complex and present a large variety of configurations, the robotic platform is presented as a modular solution which interchangeable landing gears which will be specifically designed to operate in a particular area. Then, in a first approach and after studying the problem with the end-users of this technology, it was concluded that the main scenarios to be inspected are the isolated pipes and pipe racks of different diameters. Thus, these are the main problems addressed in this work, although the system is designed to include new landing gear or perching designs for other parts of the refinery.
Last, the need of an ATEX certified system or hot-work permit to be able to operate in certain areas of the oil and gas industry imposes a mandatory requirement for the aerial robot: it must be able to immediately stop the inspection if it receives an emergency signal and fly away from the explosive environment as fast as possible. This is because, in some specific areas of these industries, such as in the proximity of gas pipes, a gas leakage could lead to an explosion in presence of any device that could generate sparks, as for example, the battery and electronics of the robot.

\section{B. Modular HYbrid Robot (MHYRO) concept}

MHYRO is a multi-modal aerial crawler robotic platform designed to solve the problem described in Section II.A. The three main requirements that should be addressed by the robot are described as follows:

1) MHYRO should be able to carry out the inspection by contact operations in the main use cases, which are isolated pipes and pipe racks.

2) The robotic platform should be able to accomplish a safe land over a pipe and be stable over it in safe conditions.

3) In case of an emergency, the robotic system should leave the inspection area as soon as possible, taking away the batteries and sensitive to explosion equipment.

Those requirements impose opposite constraints, which should be balanced in an effective way to obtain a lightweight and small inspection-ready aerial platform. As it was stated in the design of other hybrid platforms [21], a key factor in the development of these systems is the reduction of complexity. Moreover, in this case, it is proposed to include the modularity concept to address each use case interchanging the landing gear, which will be specifically designed for each inspection scenario.

MHYRO is designed to be adapted immediately before the flight taking into account the type of inspection and, after that, it is able to execute a repeated fly-crawl-fly cycle. It takes-off and flies from a specific area using a standard Xhexarotor layout configuration and controller. When it reaches the point of interest, it is able to land over the isolated pipe or pipe rack in an autonomously. Although this task could be performed by using visual servoing in the close proximity of the pipe, this paper is focused on the mechanical description and performance assessment of the aerial robot concept. Once the robot is over the pipe, it can accomplish two different tasks. On the one hand, it is able to crawl along the pipe to reach precisely the inspection area or swap between two different locomotion modes, and on the other hand, to deploy the arm with an Ultrasonic Sensor (US) to reach the measurement point in the pipe. For those tasks, it is necessary to take into account the proper requirements of the landing module installed and its controller. The scheme of the inspection action is shown in Figure 2 


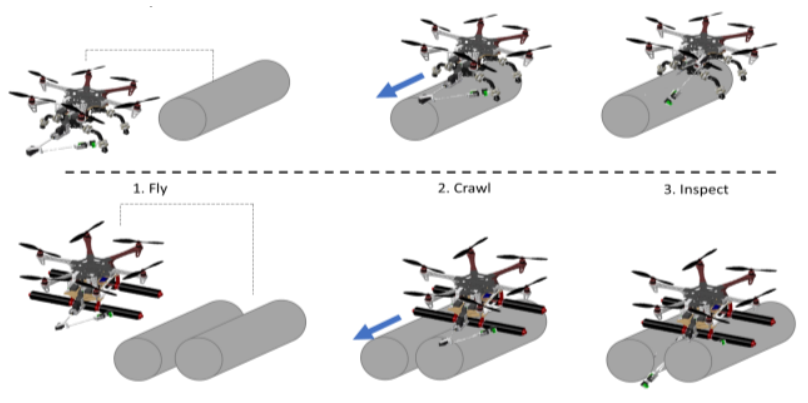

Figure 2. Concept of operation: approximation and landing on the pipe, crawling to the inspection point and inspection.

\section{MeChanicAL DESIGN}

In the mechanical design process, the aim was focused on creating an aerial robot with real inspection capabilities and functionalities. One of the most important requirements to achieve was the one related to the emergency protocol. This imposes the use of a detachable mechanism which must be rigid enough while flying but fast to deploy when the emergency protocol is triggered which must be combined with the versatility required to operate in the varied environment of an oil and gas industry. Along this section, the subsystems which compose the MHYRO prototype are presented in detail which are the aerial platform, the magnetic bridge, the robotic arm and the interchangeable landing gears or add-ons.

\section{A. Aerial Platform and Magnetic Bridge}

The aerial platform and the magnetic bridge are the core system of MHYRO. Both subsystems are the base of the modular system and, thus, have been designed to dock with the different modules that compose the robot.

This system has been built adapting a standard DJI F550 frame to replace the typical landing gear with a magnetic bridge which holds the rest of the robot. Since typical magnets only work well against traction forces, a distribution of four damped magnets has been used in such a way that they are mainly working in the axial direction. In the same way, each add-on will have a small ferromagnetic plate to maintain the subsystem in contact during normal operation. Figure 3 shows the exploded view of this prototype and a detail view of the magnetic system.

The motor selected for the aerial platform is the DJI 2312E working with the DJI 430 Lite ESC and the DJI 9540 propeller. This motorization approximately provides a nominal thrust $(50 \%)$ about 450 grams per rotor using a $4 \mathrm{~S}$ battery $(14.8 \mathrm{~V})$. The magnets used to hold the add-on are permanent electromagnets which works with a tension of 24V. In this way, a microcontroller, in this case, an Arduino Nano, is able to enable and disable them commanded by an $\mathrm{RC}$ signal. The magnetic contact interface has also been designed taking into account that it is necessary to prevent rotational, tilting and pitching movements between the ferromagnetic plate and the magnets, which is the main function of the interface shape. All these details are clearly shown in Figure 3.

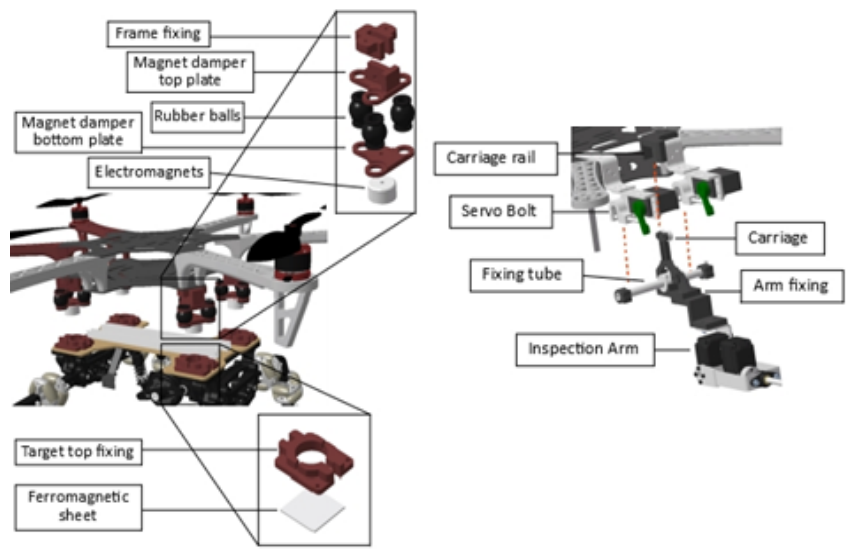

Figure 3. Magnetic bridge CAD detail and detachable arm mechanism detail

Part of the essential aerial platform is also the support of the robotic arm which must also be quick and easy to detach in manual or autonomous way in a hazardous environment. In that condition, each second counts and the time needed to retract the arm if it is deployed will not be short enough to ensure the safety during the operation.

Both, the fact that the arm is placed in the frontal part to increase the inspection workspace and the movements that it needs to accomplish the inspection, made impossible to use the same mechanism that for the landing gear. Thus, in this case, the coupling system, presented in Figure 3 consists of two components: a pair of servo actuated bolts and a carriage rail in the frame side; and the detachable arm fixing. The arm fixing has a tube which fits in both bolts, constraining all displacements but rotation along the tube axis. A carriage placed at the arm fixing fits in the aforementioned rail to prevent such rotation. This system provides accuracy during the inspection operation and prevents the arm from getting stuck when servo bolts open for releasing it.

\section{B. Lightweight Robotic Arm}

The manipulator integrated in the aerial platform has been designed for the inspection of pipe structures, allowing the access to different points of the contour, or the insertion of the end effector in the space between an array of pipes, as Figure 4 illustrates. The manipulator is a 3-DOF lightweight robotic arm in the usual upper arm-forearm configuration, with two joints at the shoulder (pitch and yaw), and one at the elbow (pitch). The arm is built with the Herkulex DRS-0101 smart servos, and a customized aluminum frame structure. Unlike our previous prototypes with compliant joints [26] designed for operating on flight, the joints of this arm are stiff to reduce the weight and because the arm is intended to operate once the aerial platform has landed, so the physical interactions will not be extremely demanding. The arm implements partially the anthropomorphic kinematics described in [27], but removing the shoulder roll joint, with the same position/trajectory control schemes based on inverse kinematics that relies on the servo controller. The main features of the arm are indicated in Table I. 


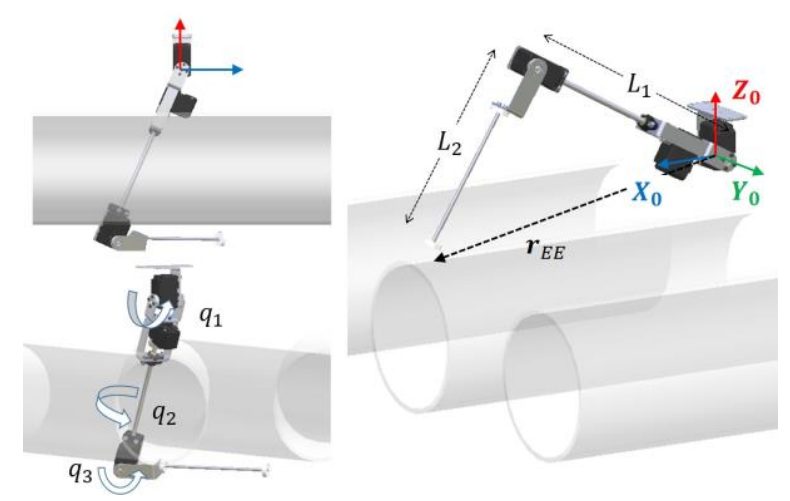

Figure 4. Different poses of the robotic arm in a scenario with pipes. Reference frame, joint variables, and link lengths.

TABLE I. MAIN FEATURES OF THE LIGHTWEIGHT ROBOTIC ARM.

\begin{tabular}{|l|l|}
\hline Weight & $0.325[\mathrm{~kg}]$ \\
\hline \multirow{2}{*}{ Dimensions } & Upper arm link: $0.25[\mathrm{~m}]$ \\
\cline { 2 - 2 } & Forearm link: $0.25[\mathrm{~m}]$ \\
\hline \multirow{3}{*}{ Rotation range } & Shoulder pitch: $\pm 120\left[{ }^{\circ}\right]$ \\
\cline { 2 - 2 } & Shoulder yaw: $\pm 120\left[{ }^{\circ}\right]$ \\
\cline { 2 - 2 } & Elbow pitch: $\pm 150\left[^{\circ}\right]$ \\
\hline Max. joint speed & $300[\% / \mathrm{s}]$ \\
\hline \multirow{2}{*}{$\begin{array}{l}\text { Lift load capacity (max. } \\
\text { gravity effect) }\end{array}$} & At elbow joint: $0.3[\mathrm{~kg}]$ \\
\cline { 2 - 2 } & At shoulder joint: $0.1[\mathrm{~kg}]$ \\
\hline
\end{tabular}

In order to facilitate the integration of an inspection sensor or device, or the wrist orientation joints, the tip of the forearm link is equipped with a Pololu aluminum shaft (4 $\mathrm{mm} \varnothing)$ with M3 threads, used as mechanical interface. Although the joints of the arm are stiff, the thin aluminum link provides a certain level of elasticity for the accommodation to the contact forces. The shoulder yaw actuator also incorporates a double flange bearing mechanism in side-by-side configuration to protect the servo against the tilting moments exerted over the upper arm.

\section{Landing Gear Modules (Add-ons)}

Two interchangeable landing gears have been designed aiming at chemical plants environments. Both have been designed to be able to land and work over two different scenarios. The first one, called Crawler-Clamp, is focused on the inspection of isolated and non-magnetic pipes, while the second one, known as Roller Add-on, will be applied to inspect racks of pipes. These are two different and complementary scenarios that can be commonly present in those industries. In fact, the Crawler-Clamp cannot operate in pipe racks since it needs a minimum clearance between the pipes to land safely on it. The same happens with the Roller Add-on, it needs at least two pipes to be stable once landed. In the following, both landing gears are detailed.

\section{1) Crawler-Clamp Add-on}

This add-on, presented in Figure 5 and Figure 6, is intended for deploying the platform on isolated pipes. It consists of two identical clamps placed on the frontal and rear side of the add-on and a central wheel.
Each clamp has two custom L-shaped rigid legs assembled through an articulated joint to a central body which houses a 2:1 reduction ratio gearbox and an HSR5980SG servo. Both legs engage the outer gears of the gearbox, so that the servo drives them symmetrically, producing the opening and closing movements of the clamp. Each leg has two omnidirectional wheels to provide contact without constraining the displacement and turn of the platform along the longitudinal axis of the pipe. The legs have been dimensioned to ensure the contact of the four omnidirectional wheels when the clamp embraces the pipe, for a strong grasping.

The central wheel is driven by a continuous rotation servo. A commercial spring damper provides compliance in the contact between the wheel and the surface when the clamps close around the pipe.

During the approach, clamps remain opened. Once a micro-switch installed at the bottom of the rear clamp detects contact, clamps close around the pipe at a constant low speed to allow a smooth attaching to the pipe. Then, the central wheel can be actuated to move the platform to the inspection point

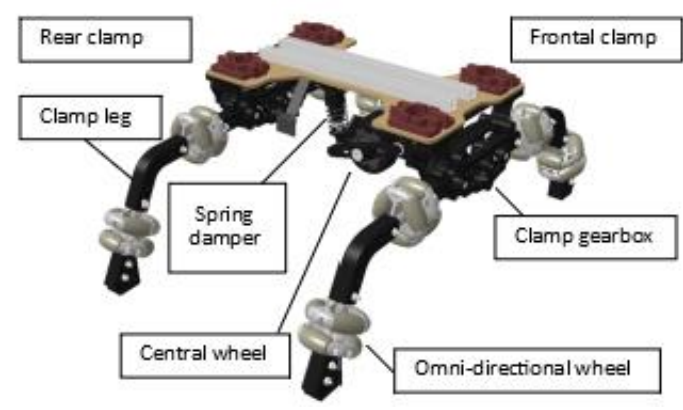

Figure 5. 3D Crawler clamp add-on (Design).

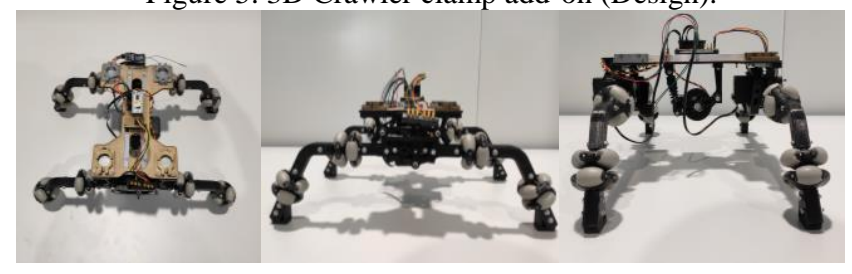

Figure 6. Crawler clamp add-on (Prototype).

TABLE II. MAIN FEATURES OF THE CRAWLER-CLAMP ADD-ON.

\begin{tabular}{|l|l|}
\hline Weight & $1.100[\mathrm{~kg}]$ \\
\hline \multirow{3}{*}{ Dimensions } & Clamp separation: $0.218[\mathrm{~m}]$ \\
\cline { 2 - 2 } & Clamp wide (opened): $0.420[\mathrm{~m}]$ \\
\cline { 2 - 2 } & Clamp height: $0.420[\mathrm{~m}]$ \\
\hline
\end{tabular}

2) Roller $\mathrm{Add}-\mathrm{On}$

Roller add-on (Figure 7 and Figure 8) is designed to perform pipe rack inspections. This add-on allows passive docking after landing, it means that the propulsion system of the aerial platform can be turned off to perform inspection tasks by the displacement of the rollers. In addition, this design does not need stabilization control, and the landing can be easily done by aligning the rollers on the pipe. 
The rolling base has a continuous rotation servo (Power HD AR-3600HB $6.7 \mathrm{kgcm}, 71 \mathrm{rpm}$ ) to provide longitudinal movement through a gear system and with a HerkuleX DRS$0602(77 \mathrm{kgcm})$ servo to control the direction by means of a crank-crank mechanism. The turning mechanism is aided by a set of ball bearings that facilitate movement. The main features of this landing gear are indicated in TABLE III.

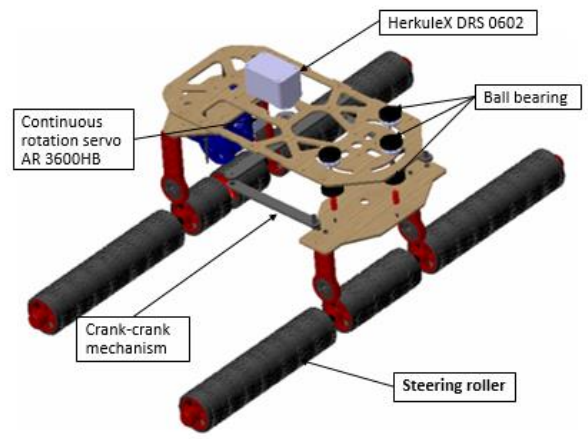

Figure 7.3D rollers add-on (Design).

The rollers have been made of a flexible material to adapt to different diameters and pipe separations. In addition, they have been designed with some roughness to achieve greater adhesion to the contact surface.

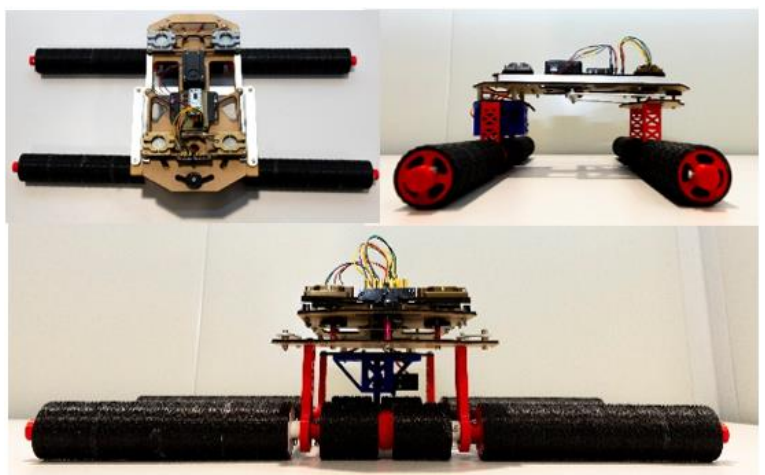

Figure 8. Rollers add-on (Prototype)

TABLE III. MAIN FEATURES OF THE ROLLER ADD-ON.

\begin{tabular}{|l|l|}
\hline Weight & $1.300[\mathrm{~kg}]$ \\
\hline \multirow{3}{*}{ Dimensions } & Roller length: $0.60[\mathrm{~m}]$ \\
\cline { 2 - 2 } & Roller separation: $0.22[\mathrm{~m}]$ \\
\cline { 2 - 2 } & Landing gear height: $0.123[\mathrm{~m}]$ \\
\hline Rotation range & $\pm 30^{\circ}$ \\
\hline
\end{tabular}

\section{Avionics and power distribution}

The aerial platform carries a Raspberry Pi 3 model $\mathrm{B}+$ as high level computer onboard powered by a power bank, a custom circuit board (magnetic bridge board) and four different batteries to supply power to the magnetic bridge, the arm and the add-ons. The propulsive system is fed by a $4 \mathrm{~S}$ battery, which also supplies power to the autopilot through a 5V UBEC. A custom detachable connector provides power and communication from the aerial platform to the add-on.
The magnetic bridge board integrates the power distribution, the magnetic bridge microcontroller, the electromagnets driver and connectors for the servo bolts.

Each add-on has a circuit board which includes the add-on microcontroller and the detachable connector plug. From this board power for the add-on RC receiver and servos is supplied, as well as power and communication for the arm. The global avionics architecture is shown in Figure 9.

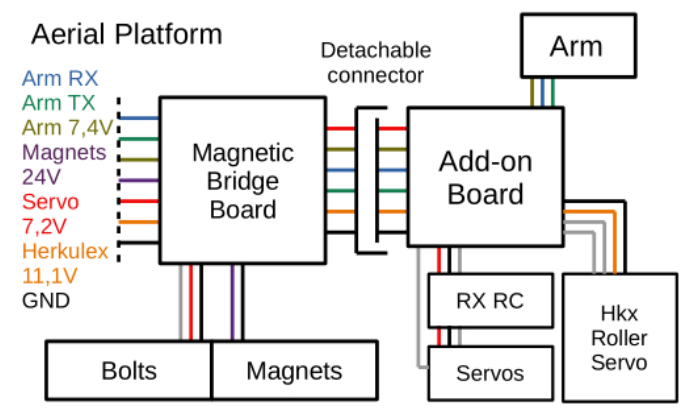

Figure 9. Power distribution

\section{CONTROL AND AUTONOMY}

The multi-modal system needs specific control strategies for the flying phase, crawling phase, inspection phase and emergency phase. Moreover, these controllers could be different depending on the crawling module installed and its morphology. Figure 10 shows the controller architecture required for our modular hybrid robot concept. The mode detection block is in charge of swapping between the four different modes and controllers depending if the robot is flying, crawling, inspecting or emergency. This mode detection module uses a combination of the sensor measurements by the inertial unit with a physical microswicth that is activated when the robot is in contact with the pipe. The emergency mode should be activated manually or automatically and independently when the gas detection sensor detects a gas leakage.

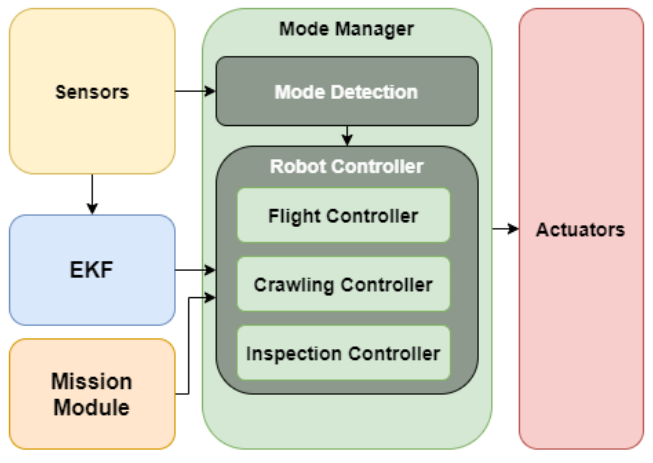

Figure 10. Controller architecture

\section{A. Flight Controller}

The flight controller used during the flying phase is based on the open-source control framework px4 [25] following the standards of an X-hexarotor layout. This includes cascade PIDs control laws which allow flying in autonomous mode closing the loop at the position level. In 
this case, when the robot is in the flying configuration, it has 4 degrees of freedoms (DoF), which are the 3D space position, $x, y$ and $z$, and the heading angle, $\psi$.

\section{B. Crawling Controller}

The crawling controller has been designed for each kind of module. The controllers are summarized below:

\section{1) Crawler clamp controller}

With this add-on, the aerial robot presents 2 DoF when it is landed on the pipe, $x_{p}$, which is the longitudinal coordinate expressed in a local reference frame along the pipe and the roll angle, $\phi$. We define $x_{p_{s p}}$ as the desired position along the pipe and $\phi_{s p}$ as the roll angle set-point over the pipe, which will be usually be $\phi_{s p}=0$. The roll angle is controlled actively using a standard cascade PID linear controller to maintain the platform stable over the pipe. Several experimental results have demonstrated that the rotors must approximately work at $20 \%$ to be able to maintain the stability over the pipe.

\section{2) Rollers controller}

As it was aforementioned, the rollers add-on provides three DoF, $x_{r p}, y_{r p}$ and $\theta_{r p}$ which represents the position and orientation of the robot in a local reference frame linked to the rack of pipes. Both depend on the thrust servo rotational speed $\omega_{r}$ and steering angle of the front roller $\gamma_{r}$. In this paper, the desired position is reached setting $\omega_{r}$ and $\gamma_{r}$ manually via $\mathrm{RC}$ in open loop. This add-on allows powering off the motors for crawling and inspection, increasing both, safety and operation time.

\section{Arm Controller}

The 3-DOF manipulator implements an inverse kinematics position/trajectory controller for the tool center point (TCP), relying on the embedded servo position controller, as described in [26] and [27]. The reference trajectory of the inspection task is transformed into a sequence of servo position references sent at $50 \mathrm{~Hz}$ at the midpoint of the trapezoidal velocity profile to achieve smooth tracking. A set of pre-defined rotation sequences is also programmed to facilitate the deployment and retrieval of the manipulator between the pipes.

\section{Emergency protocol}

Although this operation mode is not a proper control law, it must be an autonomous functionality that can be executed with total priority in case of detecting a hazardous situation. The following scheme (Figure 11) illustrates the flowchart of the protocol.

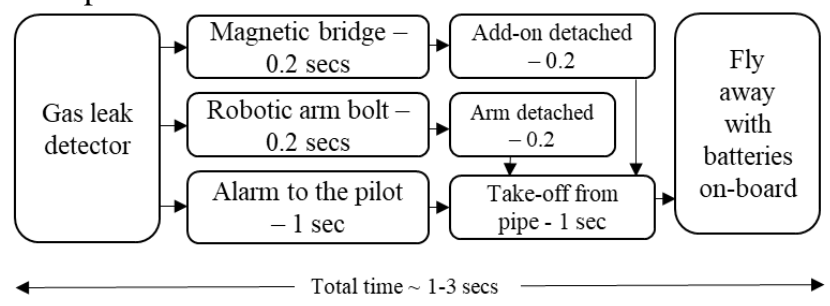

Figure 11. Emergency protocol flowchart

\section{RESUlts}

\section{A. Prototype Analysis}

The different landing gears and the robotic arm operate together to perform inspection tasks on pipes. The modular hybrid robot concept has a significant increase in mass compared to traditional multi-rotor configurations. However, this effect is compensated since the operating range of a hybrid design is significantly higher.

\section{1) Mass budget}

Figure 12 shows the mass budget of MHYRO. The hybrid design allows the landing gear to be replaced according to the inspection task to be performed. The configuration of the roller add-ons is $4.5 \%$ heavier than the crawler clamp addon, this means that the flight time is a lightly higher with the crawler-clamp installed. Both configurations have a high percentage of plastic parts (Plastic Parts and Filaflex Parts). The weight of the aerial platform constitutes half of the total weight of the modular hybrid robot. On the other hand, the robotic arm that performs the inspection and the magnetic bridge that allows the decoupling in case of emergency represent $7.4 \%$ and $12 \%$ respectively.

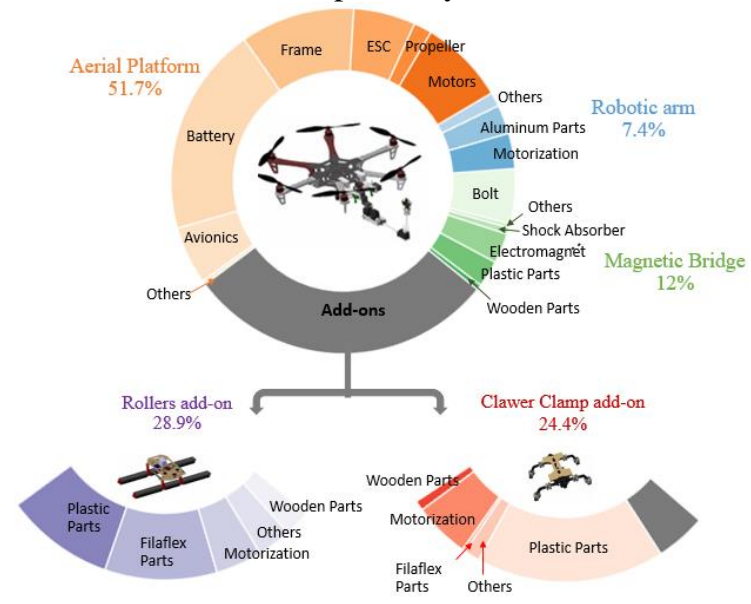

Figure 12. Mass budget

2) Add-ons capabilities comparison

The different add-ons presented along this work has been designed to cover most of the horizontal pipes of oil and gas industries. On the one hand, clamp add-on aims to inspect isolated pipes which has a clearance of at least $14 \mathrm{~cm}$. On the other hand, roller add-on is able to work in pipe racks with a maximum clearance of $40 \mathrm{~cm}$. In this way, we are able to cover most of the cases of horizontal pipes due to we are able to inspect isolated pipes and pipe racks with the same robot, just by changing the add-on installed, as it is shown in Figure 13.
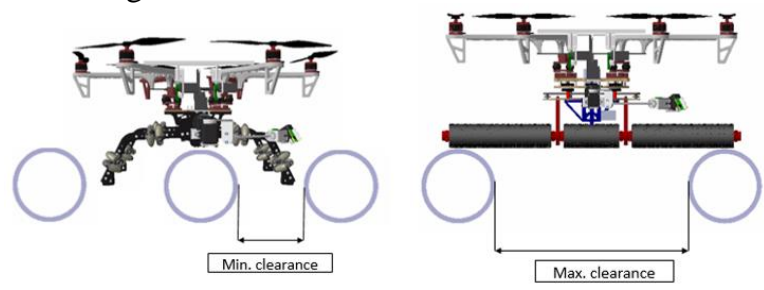

Figure 13. Add-on comparison 
3) Flight time, crawling time, inspection time and operation time

One of the most important advantages of MHYRO is the autonomy of its operation. As this system is multimodal, it is very important to detail that the full autonomy of the vehicle is a combination of their modes. Then, the following "maximum times" are defined.

$i$. Maximum flight time: It is the maximum time that the aerial robot can be lifted off and flying. This situation will be only when the robot is going to the target pipe or changing between different pipes.

ii. Maximum crawling time: It is the maximum time that the aerial robot can crawl along the pipe. It depends on the add-on mounted.

iii. Maximum inspection time: This is the maximum time that the robot can be inspecting the pipes. It depends on the add-on and the battery of the robotic arm.

iv. Maximum operation time: It is a combination of the previous times which will depend on the application scenery. In an ideal inspection, this is the sum of the total maximum times.

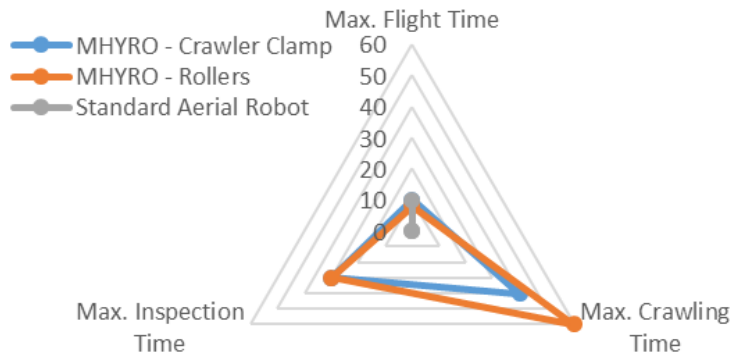

Figure 14. Operative times

Figure 14 shows a comparison of these maximum flight times of MHYRO compared with a standard multirotor. It is clear that although the robot is mainly restricted by the flight time, in this case, the multimodal configuration allows to deploy the system and work for a long time once it is landed. In this way, the operational time is much higher than the classical configuration of a flying robot which inspects and flies at the same time. An ideal operation in which all the batteries are fully consumed could last more than an hour thanks to the optimization of the different modes.

\section{B. Operation Overview}

This section presents MHYRO working in different scenarios to validate its functionalities working in isolated pipes with the crawler clamp add-on and in pipe racks with the rollers add-on. Flight and crawling maneuvers are manually controlled via RC.A video compilation of these experiments can be found in [28].

\section{1) Isolated Pipes - Crawler clamp add-on}

Figure 15 shows a sequence of images whit MHYRO landing and crawling along an isolated pipe.

In this experiment, MHYRO successfully landed over the pipe and crawled along it. After that, the inspection operation could be performed and then, the platform took off from the pipe to reach a different one or land safely on the ground.

One of the most significant results can be observed in the value of the throttle while the robot is on the pipe. In fact, as it was aforementioned, the power consumption is also lower than in hover due to the propellers are only maintaining the roll angle and does not accomplish the lifting task. Both results are shown in Figure 16.

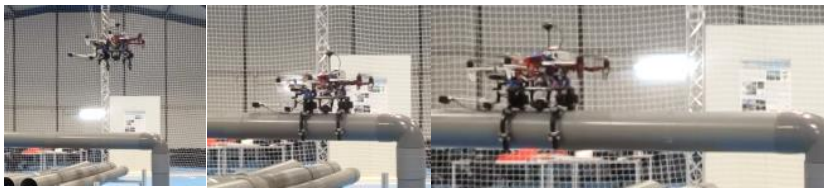

Figure 15. MHYRO landing in isolated pipes

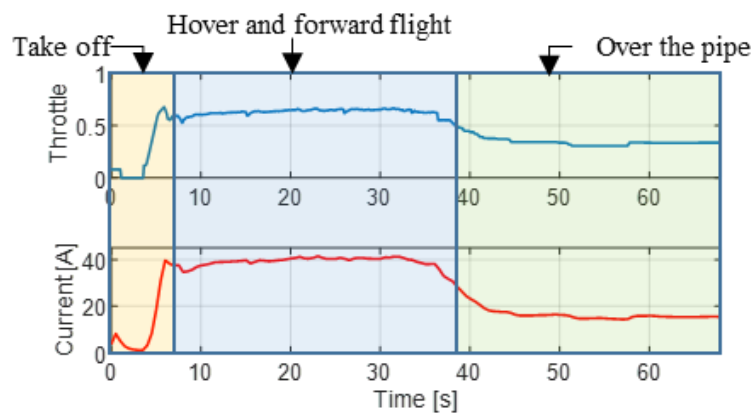

Figure 16. Throttle and current with crawler clamp

2) Pipe Rack - Rollers add-on

The second experiment was carried out to validate the rollers add-on working in a realistic scenery. Figure 17 shows how the robot is able to land over the pipe racks and in the video of [28] can be observed the crawling movement along it.

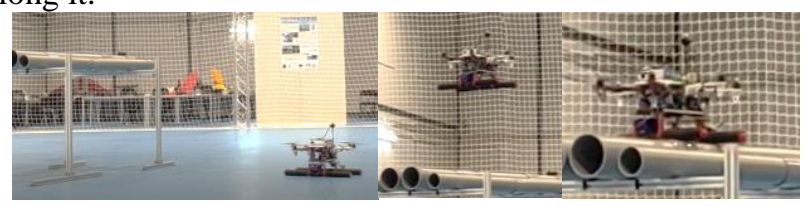

Figure 17. MHYRO landing in a pipe rack

3) Inspection phase

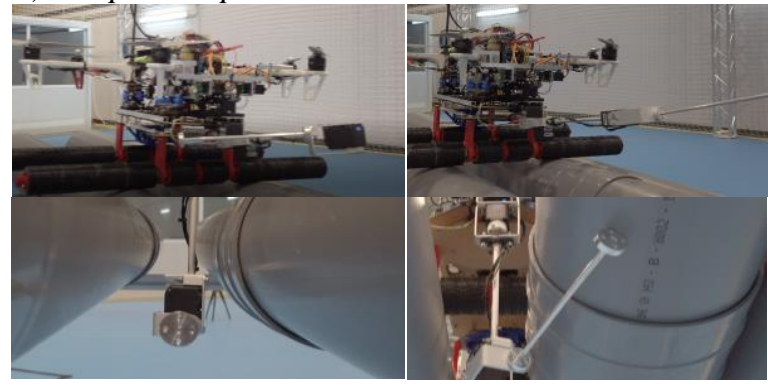

Figure 18. MHYRO deploying the robotic arm and inspecting a pipe from below

The inspection phase is divided into two sub-phases that are illustrated in Figure 18. First, the arm is deployed to be able to reach the desired workspace. Second, the endeffector establish contact with the pipe to carry out the inspection task. In this case, there is no sensor installed in 
the tip of the end effector, but the functionality is clearly presented.

\section{4) Emergency protocol}

Figure 19 presents the operation of the emergency protocol. The magnetic bridge is remotely disabled, allowing the aerial platform to leave the arm and the add-on on the pipes, and fly away carrying the batteries. This sequence of images is also presented in the video [28] in which is clearly shown how the mechanism works.

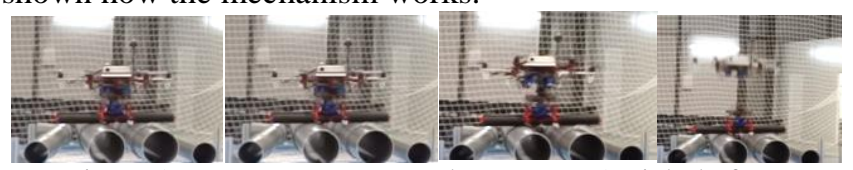

Figure 19. Emergency protocol sequence. Aerial platform detaching from the add-on and inspection arm.

\section{CONCLUSIONS}

In this paper, we have presented a new robot concept to be used in oil \& gas industries. This robot is modular and hybrid and has been designed taking into account the requirements imposed by the oil \& gas industries.

The robot presented is able to operate in the typical scenario of an oil \& gas industry in a more efficient way that the typical aerial robot which inspects and flies at the same time. Moreover, the modularity of the robot increases the application range by including new landing-gear modules or other add-ons.

Future works will include autonomous functionalities such as the development of a heterogeneous path planner which takes into account the multimodal capabilities of the robot and a final validation of the prototype in a real industry scenery out of the lab.

\section{ACKNOWLEDGMENT}

This work has been partially supported by the HYFLIERS (H20202017-779411) and AERIAL-CORE (H2020-2019-871479) projects, funded by the European Commission, the ARM-EXTEND (DPI2017-89790-R) and ARTIC (RTI2018-102224-B-I00) projects, funded by the Spanish Agencia Estatal de Investigación and the Spanish FPU program.

\section{REFERENCES}

[1] A. Ollero and B. Siciliano, Aerial Robotic Manipulation Research, Development and Applications, 129. Cham, Switz. Springer, 2019.

[2] F. Ruggiero, V. Lippiello, and A. Ollero, "Aerial manipulation: A literature review," IEEE Robotics and Automation Letters., vol. 3 (3), pp. 1957-1964, Jul. 2018.

[3] Mohiuddin, Abdullah, et al. "A Survey of Single and Multi-UAV Aerial Manipulation." Unmanned Syst. 8.2 (2020): 119-147.

[4] X. Ding, P. Guo, K. Xu, and Y. Yu, "A review of aerial manipulation of small-scale rotorcraft unmanned robotic systems," Chin. J. Aeronaut., vol. 32, no. 1, pp. 200-214, Jan. 2019.

[5] P. M. Kornatowski, S. Mintchev and D. Floreano, "An origamiinspired cargo drone," 2017 IEEE/RSJ Int. Conf. on Intelligent Robots and Systems (IROS), Vancouver, BC, 2017, pp. 6855-6862.

[6] M. Á. Trujillo, J. R. Martínez-de Dios, C. Martín, A. Viguria, and A. Ollero, "Novel aerial manipulator for accurate and robust industrial ndt contact inspection: a new tool for the oil and gas inspection industry," Sensors, vol. 19, iss. 6, p. 1305, 2019.

[7] A. E. Jimenez-Cano, J. Braga, G. Heredia and A. Ollero, "Aerial manipulator for structure inspection by contact from the underside,"
2015 IEEE/RSJ Int. Conf. on Int. Robots and Systems (IROS), Hamburg, 2015, pp. 1879-1884.

[8] M. Gschwindt, E. Camci, R. Bonatti, W. Wang, E. Kayacan and S. Scherer, "Can a Robot Become a Movie Director? Learning Artistic Principles for Aerial Cinematography," 2019 IEEE/RSJ Int. Conf. on Int. Robots and Systems (IROS), Macau, China, 2019, pp. 1107-1114.

[9] D. Yavuz, H. Akbıyı and E. Bostanc1, "Intelligent drone navigation for search and rescue operations," 2016 24th Signal Processing and Communication App. Conf. (SIU), Zonguldak, 2016, pp. 565-568.

[10] S. V. Sibanyoni, D. T. Ramotsoela, B. J. Silva and G. P. Hancke, "A 2-D Acoustic Source Localization System for Drones in Search and Rescue Missions," in IEEE Sensors Journal, vol. 19, no. 1, pp. $332-$ 341, 1 Jan.1, 2019.

[11] G. Aiello, F. Hopps, D. Santisi and M. Venticinque, "The Employment of Unmanned Aerial Vehicles for Analyzing and Mitigating Disaster Risks in Industrial Sites," in IEEE Transactions on Engineering Management.

[12] H. Guo, Q. Cui, J. Wang, X. Fang, W. Yang and Z. Li, "Detecting and Positioning of Wind Turbine Blade Tips for UAV-Based Automatic Inspection," IGARSS 2019 - 2019 IEEE International Geoscience and Remote Sensing Symposium, Yokohama, Japan, 2019, pp. 1374-1377.

[13] Sanchez-Cuevas, P. J., Ramon-Soria, P., Arrue, B., Ollero, A., \& Heredia, G. (2019). Robotic system for inspection by contact of bridge beams using UAVs. Sensors, 19(2), 305.

[14] T. Mao et al., "Development of Power Transmission Line Defects Diagnosis System for UAV Inspection based on Binocular Depth Imaging Technology," 2019 2nd Int. Conf. on Electrical Materials and Power Equipment (ICEMPE), Guangzhou, China, 2019, pp. 478-481.

[15] M. Korki, N. D. Shankar, R. Naymeshbhai Shah, S. M. Waseem and S. Hodges, "Automatic Fault Detection of Power Lines using Unmanned Aerial Vehicle (UAV)," 2019 1st Int. Conf. on Unmanned Vehicle Systems-Oman (UVS), Muscat, Oman, 2019, pp. 1-6.

[16] W. C. Myeong, K. Y. Jung, S. W. Jung, Y. H. Jung and H. Myung, "Development of a drone-type wall-sticking and climbing robot," 2015 12th International Conference on Ubiquitous Robots and Ambient Intelligence (URAI), Goyang, 2015, pp. 386-389.

[17] W. Myeong and H. Myung, "Development of a Wall-Climbing Drone Capable of Vertical Soft Landing Using a Tilt-Rotor Mechanism," in IEEE Access, vol. 7, pp. 4868-4879, 2019.

[18] H. W. Wopereis, T. D. van der Molen, T. H. Post, S. Stramigioli and M. Fumagalli, "Mechanism for perching on smooth surfaces using aerial impacts," 2016 IEEE International Symposium on Safety, Security, and Rescue Robotics (SSRR), Lausanne, 2016, pp. 154-159.

[19] Hang, Kaiyu \& Ximin, Lyu \& Song, Haoran \& Stork, Johannes \& Dollar, Aaron \& Kragic, Danica \& Zhang, Fu. (2019). Perching and resting-A paradigm for UAV maneuvering with modularized landing gears. Science Robotics. 4. eaau6637. 10.1126/scirobotics.aau6637.

[20] C. J. Dudley, A. C. Woods and K. K. Leang, "A micro spherical rolling and flying robot," 2015 IEEE/RSJ Int. Conf. on Int. Robots and Systems (IROS), Hamburg, 2015, pp. 5863-5869.

[21] R. Zufferey et al., "SailMAV: Design and Implementation of a Novel Multi-Modal Flying Sailing Robot," in IEEE Robotics and Automation Letters, vol. 4, no. 3, pp. 2894-2901, July 2019.

[22] Armanini SF, Siddall R, Kovac M, 2019, Modelling and simulation of a bioinspired aquatic micro aerial vehicle, AIAA Aviation 2019 Forum.

[23] AEROARMS Project (AErial RObotic system integrating multiple ARMS and advanced manipulation capabilities for inspection and maintenance), https://www.aeroarms.eu/

[24] AEROBI Project (AErial RObotic System for In-Depth Bridge Inspection by Contact), http://www.aerobi.eu/

[25] L. Meier, D. Honegger and M. Pollefeys, "PX4: A node-based multithreaded open source robotics framework for deeply embedded platforms," 2015 IEEE Int. Conference on Robotics and Automation (ICRA), Seattle, WA, 2015, pp. 6235-6240.

[26] A. Suarez, A. E. Jimenez-Cano, V. Vega, G. Heredia, A. RodriguezCastaño, and A. Ollero. "Design of a lightweight dual arm system for aerial manipulation" in Mechatronics, Vol. 50, 2018, pp. 30-44.

[27] A. Suarez, G. Heredia and A. Ollero, "Design of an anthropomorphic, compliant, and lightweight dual arm for aerial manipulation" in IEEE Access, vol. 6, pp. 29173-29189, 2018.

[28] Video: https://youtu.be/POu8AANqcs8 